\title{
Theophylline-7-acetic acid: lack of absorption and therapeutic effectiveness
}

\author{
J A FLEETHAM, J A OWEN, B MAY, P W MUNT, AND K NAKATSU \\ From the Departments of Medicine and Pharmacology, Faculty of Medicine, Queen's University, \\ Kingston, Ontario, Canada
}

\begin{abstract}
A double-blind cross-over trial was conducted to evaluate the effectiveness of oral theophylline-7-acetic acid (T7AA) in 13 asthmatic patients. Pulmonary function tests showed no difference between T7AA and placebo. No T7AA or theophylline was found in the sera of these patients or of healthy volunteers who took T7AA tablets or syrup.
\end{abstract}

Theophylline has been used for many years in the treatment of asthma. While it is effective, this drug is associated with therapeutic complications as evidenced by the significant incidence of adverse effects (Piafsky and Ogilvie, 1975). One of the theophylline derivatives that has been used in an attempt to circumvent side effects is theophylline7-acetic acid (T7AA), also called acepifylline and theophylline ethanoate. While this drug appears to be much less troublesome than theophylline in terms of toxicity (Phear, 1957), we were led to question its efficacy in asthmatic patients because most patients referred to the respiratory clinic at Kingston General Hospital claimed that the drug was not beneficial. We report the results of a trial in which the absorption of T7AA and its therapeutic effect were monitored.

\section{Methods and materials}

PART A

Subjects-Sixteen patients with reversible airways disease volunteered for the study and gave informed consent. Three patients developed unrelated illnesses and were forced to withdraw. Of the 13 patients (nine male, four female), two were smokers. Age range from 22 to 64 years and weight from 48 to $96 \mathrm{~kg}$. Before entering the study all patients were taking bronchodilators; eight were using methylxanthines.

Protocol-The design was double-blind, crossover with the patients taking at random T7AA or placebo capsules for one week and then the complementary capsules the second week. The volunteers were told not to alter normal daily routine except to take the capsules four times daily; those previously on methylxanthines were asked to stop four days before and for the duration of the study. They also kept a daily record of air- $\frac{\otimes}{\not}$ flow obstruction on a portable ventilatory function $\stackrel{\square}{\rightarrow}$ indicator Airflowmeter (Allen and Hanbury) (Friedman and Walker, 1975). On the seventh morning of each week, a fasting blood sample was drawn at 0830 from each patient after which the final capsule was taken. An interview and spirometric testing followed. During the interview patients gave subjective evaluations of their ease of breathing and reported any adverse effects. $\frac{3}{3}$ Spirometry was recorded using a 13.5L Respirometer (Warren E Collins, In). At 1130 a second $\frac{9}{3}$ blood sample was taken; these samples were centrifuged, and the plasma was frozen until assayed. Samples were assayed for theophylline and $\frac{7}{2}$ T7AA by the method of Owen and Nakatsu (1978); for both drugs the limits of sensitivity o were $0.5 \mu \mathrm{g} / \mathrm{ml}$.

PART B

Ten healthy volunteers each took two tablets of T7AA (Dynaphylline, $250 \mathrm{mg}$, Welcker-Lyster) $\stackrel{\varrho}{\mathrm{C}}$ from recently acquired lots. For eight subjects $\stackrel{\oplus}{\odot}$ pre-drug control and two-hour blood samples were $?$ taken; for two subjects control, one-, two-, three-, $\frac{0}{0}$ and four-hour samples were taken. Plasma samples $\overrightarrow{\mathbb{D}}$ were assayed for theophylline as above and by high $\frac{O}{\mathbb{D}}$ pressure liquid chromatography (Nakatsu et al, 1978), and for T7AA as above.

A further six normal volunteers received $25 \mathrm{ml} \varrho$ 
of T7AA containing syrup (Dynaphylline, $20 \mathrm{mg} / \mathrm{ml}$, Welcker-Lyster). Blood samples taken were pre-drug control, two hours and four hours. These were assayed for theophylline and T7AA as above.

\section{Results}

The data obtained in Part $\mathrm{A}$ of this study are summarised in the table. Patients reported no subjective differences between the two treatments. The home respiratory performance estimates with the Airflowmeter were not different from one week to the next. The spirometric measurements in the laboratory corresponded with these observations and showed no significant improvement in forced vital capacity, $\mathrm{FEV}_{1}$, and MMF when patients were taking T7AA compared to placebo.

No T7AA or theophylline was found in any of the patients' plasma samples. These data are supported by the data of Part B obtained from the 10 healthy volunteers who took T7AA tablets. Again, no T7AA or theophylline was found in any sample at one, two, three, or four hours after the tablets were taken. In the six normal volunteers who received T7AA syrup no T7AA or theophylline was detected in their plasma samples at any time after the drug was ingested.

Part A: Comparison of theophylline-7-acetic acid and placebo in 13 patients

\begin{tabular}{lcc}
\hline & T7AA & $\begin{array}{c}\text { Lactose } \\
\text { placebo }\end{array}$ \\
\hline Subjective response & $-0 \cdot 5$ & $-0 \cdot 4$ \\
Airflowmeter (units) & $-11 \pm 1 \cdot 3$ & $-11 \pm 5$ \\
Forced vital capacity (ml) & $0 \pm 88$ & $-130 \pm 87$ \\
Forced expiratory volume & $-83 \pm 113$ & $-236 \pm 155$ \\
1 second (ml) & & \\
Maximal mid-expiratory flow (ml/sec) & $-108 \pm 123$ & $-255 \pm 188$ \\
Plasma T7AA ( $\mu \mathrm{g} / \mathrm{ml})$ at & 0 & 0 \\
$\quad 0830$ & 0 & 0 \\
$\quad 1100$ & 0 & 0 \\
Plasma theophylline $(\mu \mathrm{g} / \mathrm{ml})$ at & 0 & 0 \\
$\quad 0830$ & 0 & \\
\hline
\end{tabular}

All values are expressed as mean of data from 13 patients \pm SEM. None of the pairs of data was significantly different. Subjective response was graded in comparison to pretrial condition as follows: +3 pronounced improvement; +2 moderate improvement; +1 mild improvement; 0 no change; -1 mild worsening; -2 moderate worsening; and -3 pronounced worsening.

\section{Discussion}

The pulmonary function studies and the patients' personal reports indicated that T7AA was not significantly more beneficial than placebo. These observations are consistent with plasma analysis that showed no T7AA at any of the sampling times.

Since possibly the lack of therapeutic effect and lack of appearance of drug in the plasma could have been due to patient non-compliance, T7AA was administered under observation to 10 healthy volunteers who were known by the investigators to be reliable. The data obtained from these samples supported those collected from patient samples. Thus it appeared that the drug was not being absorbed by either the patients or the healthy volunteers.

It was still possible that T7AA was not absorbed because of the way in which the tablet was manufactured with poor release into an absorbable form. This seems highly unlikely in view of the finding that no T7AA was found in plasma samples of volunteers who had ingested T7AA syrup. Thus it is evident that T7AA is not effective because it is not absorbed from the gastrointestinal tract.

This interpretation is consistent with the theoretical consideration of Wagner (1975) and the experimental observations of Strolin-Benedetti et al (1971) and Zuidema and Merkus (1978). T7AA has an octanol: water partition coefficient of $2.7 \times 10^{-4}$; according to the theoretical model of Wagner (1975) the drug would be only very slowly absorbed with an absorption t $\frac{1}{2}$ of about 1700 hours. Strolin-Benedetti et al (1971) administered large oral doses $(1.2 \mathrm{~g} / \mathrm{kg})$ of ${ }^{14} \mathrm{C}$ labelled T7AA to rats and found only small amounts of radioactivity in plasma or urine. Zuidema and Merkus (1978) reported a similar lack of absorption of T7AA in a patient who had been taking the drug for months. On the basis of their observations and those reported here it seems wise to avoid T7AA and to use other methylxanthine preparations to treat asthmatic patients.

We have noted that many doctors do not appreciate that T7AA is unlike the theophylline salts and is a chemical derivative of theophylline. As such it cannot dissociate into theophylline and acetic acid; the lack of any theophylline in serum of individuals taking T7AA reinforces this point.

Supported by the Ontario Respiratory Disease Foundation.

\section{References}

Friedman, M, and Walker, S (1975). Assessment of lung function using an air-flow meter. Lancet, 1, $310-311$.

Nakatsu, K, Owen, J A, and Scully, K (1978). Reliable 
fifteen-minute assay for theophylline. Clinical Biochemistry, 11, 148-149.

Owen, J A, and Nakatsu, K (1978). Spectrophotometry of theophylline-7-acetic acid and theophylline. Clinical Chemistry, 24, 367-368.

Phear, D N (1957). An objective comparison of two recent neutral theophylline compounds with aminophylline. British Medical Journal, 2, 74-75.

Piafsky, K M and Ogilvie, R I (1975). Dosage of theophylline in bronchial asthma. New England Journal of Medicine, 292, 1218-1222.

Strolin-Benedetti, M, Larue, D A, Oliver, C, and
Bouvet, P (1971). Absorption and excretion of piperazine theophylline ethanoate $-{ }^{14} \mathrm{C}$ in the rat. Arzneimittel-Forschung, 21, 662-664.

Wagner, J C (1975). Fundamentals of Clinical Pharmacokinetics, p 217. Drug Intelligence Publications, Hamilton.

Zuidema, J, and Merkus, F W (1978). Is acephylline a theophylline bronchodilator? Lancet, 1, 1318-1319..

Requests for reprints to: Dr K Nakatsu, Department of Pharmacology, Faculty of Medicine, Queen's University, Kingston, Ontario, K7L 3N6. 